Check for updates

Cite this: Mater. Adv., 2022, 3, 1254

Received 10th November 2021 Accepted 29th November 2021

DOI: 10.1039/d1ma01052b

rsc.li/materials-advances

\title{
Near-infrared absorbing aza-BODIPYs with 1,7-di-tert-butyl groups by low-barrier rotation for photothermal application $\dagger$
}

\author{
Sicheng Li, $\ddagger^{a}$ Meiheng LV, $\ddagger^{\text {ab }}$ Jie Wang, $\stackrel{\ddagger}{c}^{c}$ Dongxiang Zhang, $\ddagger^{a}$ Zhangrun Xu (D) ${ }^{c}$ \\ and Xin-Dong Jiang (D) *a \\ Near-infrared absorbing aza-BODIPYs with 1,7-di-tert-butyl groups (tBuazaBDP) were successfully \\ synthesized. Based on X-ray crystal analysis, S-tBuazaBDP is found to be more twisted than the \\ reported dye tetraphenyl aza-BODIPY. tBuazaBDPs possess excellent optical properties such as high \\ molar extinction coefficients, broad full width at half maxima, and large Stokes shifts. Experimental \\ results and theoretical calculations proved that the distal $-t \mathrm{Bu}$ groups in S-tBuazaBDP can undergo a \\ low-barrier rotation. Although this low-barrier rotation of the distal - $t$ Bu groups in tBuazaBDP leads to \\ a low quantum yield, the photothermal conversion efficiency and the singlet oxygen generation ability of \\ S-tBuazaBDP-NPS are obviously enhanced, which are highly desirable for a photothermal therapy agent.
}

\section{Introduction}

Since azadipyrromethene was first reported in 1943 by M. Rogers, aza-BODIPYs have been attracting increasing attention. ${ }^{1-3}$ Owing to their near-infrared (NIR) absorption, high molar extinction coefficient and adjustable wavelength, aza-BODIPYs are widely applied in NIR dyes, fluorescent chemosensors, sensitized solar cells, photodynamic therapy (PDT), photothermal therapy (PTT) and so forth. ${ }^{4-10}$ All these applications directly reflect one or more of three pathways they follow to return to the ground state from the excited state, that is, emitting photons (fluorescence), following non-radiative relaxation pathways (heat generation), or exiting from the singlet to the triplet state (T1) via intersystem crossing (ISC) to generate singlet oxygen with oxygen. ${ }^{11-15}$ Recently, PTT and PDT, which correspond to the latter two pathways, have aroused widespread interest in the phototherapeutic field. Therefore, the closing of the fluorescent channel is key to establishing their application for PTT and/or PDT. ${ }^{16-23}$

\footnotetext{
${ }^{a}$ Liaoning \& Shenyang Key Laboratory of Functional Dye and Pigment, Shenyang University of Chemical Technology, Shenyang, 110142, China. E-mail: xdjiang@syuct.edu.cn

${ }^{b}$ State Key Laboratory of Molecular Reaction Dynamics, Dalian Institute of Chemical Physics, Chinese Academy of Sciences, Dalian 116023, P. R. China ${ }^{c}$ Department of Chemistry, College of Sciences, Northeastern University, Shenyang 110819, China

† Electronic supplementary information (ESI) available. CCDC 2117398. For ESI and crystallographic data in CIF or other electronic format see DOI: 10.1039/d1ma01052b \$ These authors contributed equally to this work.
}

Compared with PDT, PTT has the advantage of not being restricted by the tumor hypoxic environment. To enhance photothermal conversion efficiency, first of all, it is necessary to select a NIR light. High tissue penetration requires a photothermal transduction agent (PTA) for NIR absorption, enhancing light absorption, and decreasing light scattering. ${ }^{24-26}$ Enhancement of energy conversion is achieved by the charge induced effect or the rotation of the bulk groups (such as $-\mathrm{CF}_{3}$ ) for nonradiative decay. ${ }^{27-29}$ On the one hand, intramolecular photoinduced electron transfer is successfully established between the dimethylamino unit and the dye, contributing largely to the enhanced photothermal conversion efficiency (PCE) via nonradiative decay of the excited photons. ${ }^{30,31}$ Based on theoretical calculations and steady-state and time-resolved photoluminescence spectra, the intramolecular photoinduced electron transfer was proved to increase the probability of the excited photons to follow a nonradiative decay path for enhancing photothermal conversion. ${ }^{32-35}$ On the other hand, the rotational mode can also promote efficient nonradiative decay, that is, by enhancing the conversion of light into heat. For example, Peng et al. reported a NIR light-driving barrier-free group rotation $\left(-\mathrm{CF}_{3}\right)$ with high photothermal conversion efficiency. ${ }^{36-39}$ Therefore, when a PTA absorbs light, heat and/ or cytotoxic ROS can be produced via non-radiative decay or intersystem crossing (ISC) for PTT and/or PDT to induce apoptosis or necrosis of tumor cells. ${ }^{40-43}$ So, the temperature of the tumor tissue surrounding the PTA rapidly increases $\left(>50{ }^{\circ} \mathrm{C}\right.$ ) and achieves tumor ablation. ${ }^{44-47}$ Most inorganic nanomaterials are known to usually suffer from the defects of poor biodegradability and heavy metal leakage, which hinder 

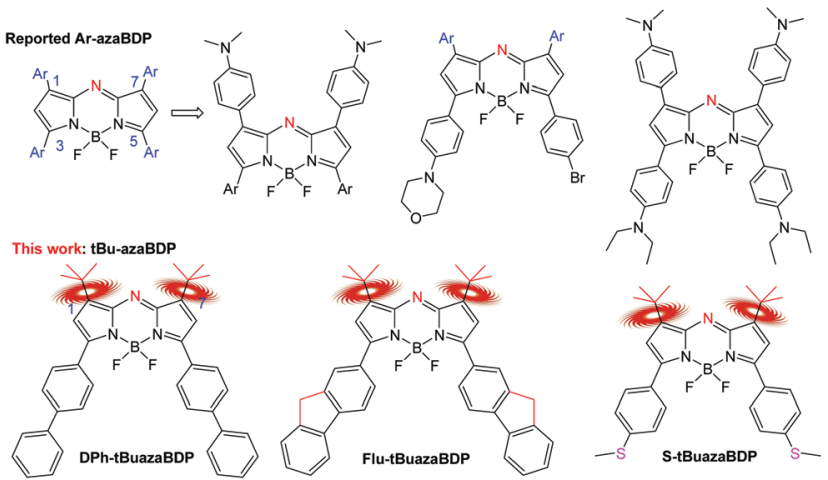

Fig. 1 Design strategies for tBuazaBDPs with the $-t$ Bu groups in 1,7positions.

their comprehensive application. ${ }^{4-51}$ In contrast, organic phototherapy reagents are found to show prominent biodegradability and low toxicity in phototherapy. ${ }^{52-58}$ Some literatures about organic phototherapy reagents have reported to enhance nonradiative decay pathways. $^{59-61}$ The traditional organic dye aza-BODIPY displays more favorable red-shifted absorbance compared with the common organic phototherapy reagents like porphyrin and phthalocyanine derivatives. ${ }^{62,63}$ Furthermore, aza-BODIPYs have high molar extinction coefficients in the NIR region with good photostability, and are therefore promising candidates for tumor theranostics. For example, the groups of Huang, Dong, Zhao and others have reported valuable structures related to " $\mathrm{NMe}_{2}$-bearing" aza-BODIPY derivatives (Fig. 1). ${ }^{64-66}$ In their systems, the dialkylamino group caused photoinduced electron transfer, which quenched the fluorescence of aza-BODIPYs, resulting in the creation of a PTT effect. Moreover, when combining PTT and PDT effects, highly efficient therapeutics was obtained. ${ }^{67,68}$ However, based on this mechanism of the charge induced effect, only 1,7,3,5-tetra-aryl aza-BODIPY-based PTAs in the aza-BODIPY system were reported to act as PTT/PDT synergetic agents for anticancer. Therefore, a highly efficient NIR aza-BODIPYbased phototherapy agent is still desired and remains to be developed. Up to now, only two examples of non-aryl substituted aza-BODIPY structures have been reported. ${ }^{69,70}$ So, the unique spectral properties and rules of non-aryl substituted aza-BODIPY dyes are still unknown, and the new applications of the functional enhancement are not clear. Our previous work on aza-BODIPYs paved the way to the discovery of non-aryl substituted azaBODIPYs. ${ }^{71-74}$ Herein, a novel non-aryl substituted aza-BODIPY, that is, aza-BODIPY with the $-t \mathrm{Bu}$ groups at 1,7-positions (tBuazaBDP), was skillfully constructed to undertake an original research work (Fig. 1). Since the rotation of the bulk groups for nonradiative decay can promote energy conversion, tBuazaBDP as a promising platform is further explored for photothermal conversion and singlet oxygen generation in this work.

\section{Experimental}

All the chemicals and solvents used in this work were of analytical grade and were purchased from Energy Chemical \& Technology (Shanghai) Co. Ltd and used without further purification. ${ }^{1} \mathrm{H}$ NMR spectra were recorded on a VARIAN Mercury 400 or $500 \mathrm{MHz}$ spectrometer. ${ }^{1} \mathrm{H}$ NMR chemical shifts $(\delta)$ are given in ppm downfield from $\mathrm{Me}_{4} \mathrm{Si}$, determined by residual chloroform $(\delta=7.26 \mathrm{ppm}) \cdot{ }^{13} \mathrm{C}$ NMR spectra were recorded on a VARIAN Mercury $125 \mathrm{MHz}$ spectrometer in $\mathrm{CDCl}_{3}$; all signals are reported in ppm with the internal chloroform signal at $\delta 77.0 \mathrm{ppm}$ as the standard. Fluorescence spectra were recorded on an F-4600 spectrophotometer and are reported in $\mathrm{cm}^{-1}$. UV/Vis spectra were recorded on a UV-2550 spectrophotometer at room temperature. The refractive index of the medium was measured by using a 2 W Abbe's refractometer at $20{ }^{\circ} \mathrm{C}$.

The fluorescence quantum yields $\left(\Phi_{\mathrm{f}}\right)$ of the aza-BODIPY systems were calculated using the following relationship (eqn (1)):

$$
\Phi_{\mathrm{f}}=\Phi_{\text {ref }} F_{\text {sampl }} A_{\text {ref }} n_{\text {sampl }}^{2} / F_{\text {ref }} A_{\text {sampl }} n_{\text {ref }}^{2}
$$

Here, $F$ denotes the integral of the corrected fluorescence spectrum, $A$ is the absorbance at the excitation wavelength, $n$ is the refractive index of the medium, and ref and sampl denote parameters from the reference and unknown experimental samples, respectively.

The reference system used was aza-BODIPY as the standard $\left(\Phi_{\mathrm{f}}=0.36 \text { in chloroform, } \lambda_{\mathrm{abs}}=688 \mathrm{~nm}\right)^{14}$ for $\mathbf{S} / \mathbf{D P h} /$ Flu-tBuazaBDP.

Crystals suitable for X-ray structure determination were mounted on a Mac Science DIP2030 imaging plate diffractometer and irradiated with graphite monochromated Mo-K $\alpha$ radiation $(\lambda=0.71073 \AA)$ for data collection. The unit cell parameters were determined by separately auto-indexing several images in each data set using the DENZO program (MAC Science). ${ }^{75}$ For each data set, the rotation images were collected in $3^{\circ}$ increments with a total rotation of $180^{\circ}$ about the $\varphi$ axis. The data were processed using SCALEPACK. The structures were solved by a direct method with the SHELX-97 program. ${ }^{76}$ Refinement on $F^{2}$ was carried out using the full-matrix leastsquares method with the SHELX-97 program. All non-hydrogen atoms were refined using the anisotropic thermal parameters. The hydrogen atoms were included in the refinement along with the isotropic thermal parameters.

All theoretical calculations of this work were performed based on density functional theory (DFT) and time-dependent density functional theory (TDDFT) methods via the Gaussian 16 program suite. ${ }^{77}$ Becke's three-parameter hybrid exchange function with the Lee-Yang-Parr gradient-corrected correlation functional augmented by Grimme's D3-dispersion correction with the Becke-Johnson damping function (B3LYP-D3BJ) and the 6-31G(D) basis set were selected for the calculations. And the solvation effects of dichloromethane were also taken into consideration with the solvation model based on density (SMD).

The singlet oxygen $\left({ }^{1} \mathrm{O}_{2}\right)$ quantum yields of DPh-tBuazaBDP, Flu-tBuazaBDP and S-tBuazaBDP were determined by the equation $\Phi_{\Delta}=\Phi_{\Delta \mathrm{st}} \times\left(S / S_{\mathrm{st}}\right) \times\left(F_{\mathrm{st}} / F\right) \times\left(\mathrm{PF}_{\mathrm{st}} / \mathrm{PF}\right)$, where $S$ is the slope of the change in the absorbance of DPBF at the absorbance 
maxima with the irradiation time. $F$ is the absorption correction factor, which is given as $F=1-10^{-\mathrm{A}}$, and $\mathrm{PF}$ is the absorbed photonic flux ( $\mu$ Einstein $\mathrm{dm}^{-3} \mathrm{~s}^{-1}$ ). Singlet oxygen yield with 2,6-diiodobodipy $\left(\Phi_{\Delta}=0.85\right.$ in toluene) was used as the standard. The optical power density was measured by using a CEL-NP 2000 power meter.

The nanoparticles of Flu-tBuazaBDP and S-tBuazaBDP were prepared by nanoprecipitation with F127. A mixture of F127 (5 mg) and S-tBuazaBDP ( $2 \mathrm{mg}$ ) was dissolved in THF (1 ml). The solution was subjected to ultrasound for $1 \mathrm{~min}$. Then, $200 \mu \mathrm{L}$ of this solution was injected into distilled water $(10 \mathrm{ml})$ with ultrasound at room temperature. The mixture was stirred overnight for the removal of THF. Subsequently, the desired S-tBuazaBDP-NPs could be obtained by centrifugation at $6000 \mathrm{rpm}$ for $5 \mathrm{~min}$. With the same procedure, Flu-tBuazaBDP-NPs were prepared.

Photothermal conversion efficiency calculation. For the purpose of evaluating the photothermal ability of S-tBuazaBDP-NPS and Flu-tBuazaBDP-NPs, the effect of solution on conversion efficiency was discussed. S-tBuazaBDP-NPs or Flu-tBuazaBDPNPs were irradiated by a $690 \mathrm{~nm}$ laser at a power density of $0.8 \mathrm{~W} \mathrm{~cm}^{-2}$. The temperature changes were monitored by using a FLIR-1910582 thermal camera. S-tBuazaBDP-NPs or Flu-tBuazaBDP-NPs at $20 \mu \mathrm{M}$ concentration were prepared and irradiated with a $690 \mathrm{~nm}$ laser $\left(0.8 \mathrm{~W} \mathrm{~cm}^{-2}\right)$ for $5 \mathrm{~min}$. The temperature changes were monitored during irradiation. To measure the photothermal conversion efficiency, S-tBuazaBDP-NPs or Flu-tBuazaBDP-NPs were exposed to $690 \mathrm{~nm}$ irradiation at $0.8 \mathrm{~W} \mathrm{~cm}^{-2}$ for $5 \mathrm{~min}$, and then the solution was cooled down to room temperature. The temperature of the solution was recorded at an interval of $30 \mathrm{~s}$ during this process.

The photothermal conversion efficiency $(\eta)$ was measured according to the reported method:

$$
\eta=\left(\mathrm{hs}\left(T_{\max }-T_{\text {surr }}\right)-Q_{\text {Dis }}\right) /\left(I \times\left(1-10^{-\mathrm{A} 690}\right)\right)
$$

$h$ is the heat transfer coefficient and $s$ is the surface area of the container. $Q_{\text {Dis }}$ represents heat dissipated from the laser mediated by the solvent and container. For both S-tBuazaBDPNPs and Flu-tBuazaBDP-NPs, $I$ is the laser power and $A$ is the absorbance at $690 \mathrm{~nm}$.

$$
\mathrm{hs}=m C_{\mathrm{H} 2 \mathrm{O}} / \tau_{\mathrm{s}}
$$

$m$ is the mass of the solution containing the photoactive material, $C$ is the specific heat capacity of the solution $\left(C_{\mathrm{H}_{2} \mathrm{O}}=4.2 \mathrm{~J} \mathrm{~g}^{-1}{ }^{\circ} \mathrm{C}\right)$, and $\tau_{\mathrm{s}}$ is the associated time constant.

$$
T=-\tau_{\mathrm{s}} \ln (\theta)
$$

$\theta$ is a dimensionless parameter, known as the driving force temperature.

$$
\theta=\left(T-T_{\text {surr }}\right) /\left(T_{\max }-T_{\text {surr }}\right)
$$

$T_{\max }$ and $T_{\text {surr }}$ are the maximum steady state temperature and the environmental temperature, respectively.
For the live and dead cell staining tests, human colon cancer cells (LoVo) were seeded onto $35 \mathrm{~mm}$ confocal dishes for $24 \mathrm{~h}$, and then the cells were subjected to the following treatments: Control, where cells were incubated without any treatments; Control + Light, where cells were irradiated with a $635 \mathrm{~nm}$ laser $\left(10 \mathrm{~mW} \mathrm{~cm}^{-2}\right)$ for $20 \mathrm{~min}$ followed by incubation with $20 \mu \mathrm{M}$ S-tBuazaBDP-NPs at $37{ }^{\circ} \mathrm{C}$ for $4 \mathrm{~h}$; S-tBuazaBDP-NPs + Light, where cells were incubated with $20 \mu \mathrm{M}$ S-tBuazaBDP-NPs at $37{ }^{\circ} \mathrm{C}$ for $4 \mathrm{~h}$, followed by $635 \mathrm{~nm}$ laser irradiation $\left(10 \mathrm{~mW} \mathrm{~cm}^{-2}\right)$ for $20 \mathrm{~min}$. After incubation for $4 \mathrm{~h}$, cells were stained with the Calcein AM/PI Apoptosis Detection Kit according to the manufacturer's instructions, and imaged by confocal laser scanning microscopy (Olympus FV-3000). Calcein AM was excited with a $488 \mathrm{~nm}$ laser, and detected in the range from 500 to $540 \mathrm{~nm}$; PI was excited with a $488 \mathrm{~nm}$ laser, and detected in the range from 650 to $690 \mathrm{~nm}$.

Full information on synthesis, NMR, HRMS and so forth is provided in the ESI. $\dagger$

\section{Results and discussion}

Using 3,3-dimethylbutan-2-one, aza-BODIPYs with the $-t \mathrm{Bu}$ groups at 1,7-positions (tBuazaBDPs) were prepared (Scheme 1). 4,4-Dimethyl-1-(thiophen-2-yl)pent-2-en-1-one was obtained by aldol condensation. After that, nitro derivatives of chalcones were synthesized by using 1,4-Michael addition. Then, by the formation of a bridge between an imino group and the complex of $\mathrm{BF}_{3} \cdot \mathrm{Et}_{2} \mathrm{O}$, the target dyes tBuazaBDPs with the $-t \mathrm{Bu}$ groups at 1,7-positions were obtained. NMR and HRMS spectra of them further verified their formulation. Moreover, the structure of S-tBuazaBDP was distinctly confirmed by X-ray crystal analysis (Fig. 2). The C1-C23 bond (1.507 $\AA$ ) in S-tBuazaBDP is shorter than the classical C-C bond (1.54 $\AA$ ) of alkanes. The $\mathrm{sp}^{3}$ hybridized boron center in S-tBuazaBDP appeared in a distorted tetrahedral geometry with the angles $\mathrm{N} 1-\mathrm{B} 1-\mathrm{N} 2$ of $105.5^{\circ}$ and $\mathrm{F} 1-\mathrm{B} 1-\mathrm{F} 2$ of $110.0^{\circ}$, which deviated from the ideal value of $119.5^{\circ}$. Surprisingly, the C2-C3-N1-B1 dihedral angles were $170.1^{\circ}$ and deviated from the ideal value 180 , indicating that the boron atom more upwarped from the core structure of S-tBuazaBDP (Fig. 2b). Especially, the C23-C1C4-N3 dihedral angles were $8.2^{\circ}$ and the C16-C13-C12-N3

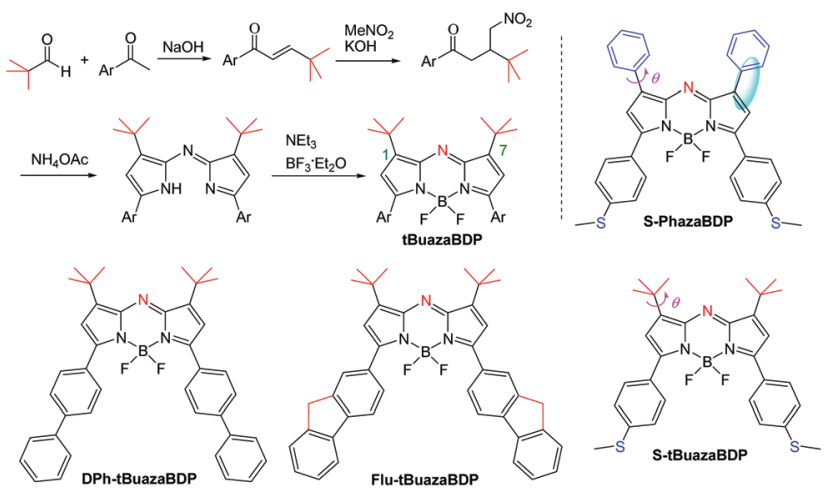

Scheme 1 Synthesis of 1,7-di-tert-butyl substituted tBuazaBDPs. 


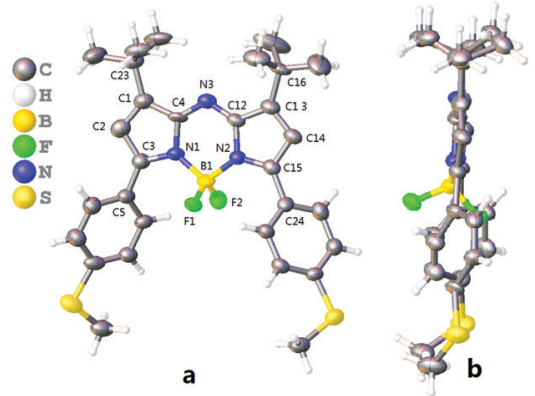

Fig. 2 ORTEP views of S-tBuazaBDP (CCDC: 2117398). (a) Front and (b) side views of the molecular structures. The $C, H, B, N, F$ and $S$ atoms are depicted with thermal ellipsoids set at the $30 \%$ probability level.

dihedral angles were $3.7^{\circ}$; the two $-t$ Bu groups in S-tBuazaBDP were located at both sides of the core (Fig. 2b). In brief, the molecular structure of S-tBuazaBDP was severely twisted.

Since the $-t \mathrm{Bu}$ group was directly introduced into the parent nucleus of aza-BODIPY, we were curious to gain insight into the spectral properties (Fig. 3 and Table 1). Compared to the reported dye S-PhazaBDP with the phenyl groups at 1,7positions $\left(\lambda_{\mathrm{abs}} / \lambda_{\mathrm{em}}=702 / 746 \mathrm{~nm}, \Phi_{\mathrm{f}}=0.06\right)$ (Scheme 1 and Table 1$),{ }^{78}$ the maxima of the absorption $(666 \mathrm{~nm})$ and fluorescence spectra $(713 \mathrm{~nm})$ of S-tBuazaBDP were indeed blueshifted by $36 \mathrm{~nm}$ and $33 \mathrm{~nm}$ respectively, but were still located at the NIR region. These results are mainly due to the reduction of the conjugated system, when replacing $-\mathrm{Ph}$ with the $-t \mathrm{Bu}$ group. And, its fluorescence quantum yield was found to be reduced to 0.04 , and the molar extinction coefficient was also reduced to $85000 \mathrm{M}^{-1} \mathrm{~cm}^{-1}$, owing to the ICT effect of the electron-rich methylthioether substituents and non-radiative relaxation offered by the rotation of the $-t \mathrm{Bu}$ group. Although the spectral properties have some attenuation after the $-t \mathrm{Bu}$ substitution, they are still in the tolerance range, which is comparable to those of the classical dyes BODIPYs or rhodamines. $^{2,3} \quad$ Especially, 1,7-di-tert-butyl substituted S-tBuazaBDP showed a larger Stokes shift (SS: $47 \mathrm{~nm})$ and a broader full width at half maxima (FWHM: $73 \mathrm{~nm}$ ), compared to those of S-PhazaBDP (SS: $43 \mathrm{~nm}$; FWHM: $52 \mathrm{~nm}$ ). Taken together, the large Stokes shift for S-tBuazaBDP may be indicative of significant differences between the geometry of S-tBuazaBDP in its $S_{0}$ and $S_{1}$ states, leading to an unfavorable Franck-Condon factor. In addition, compared to
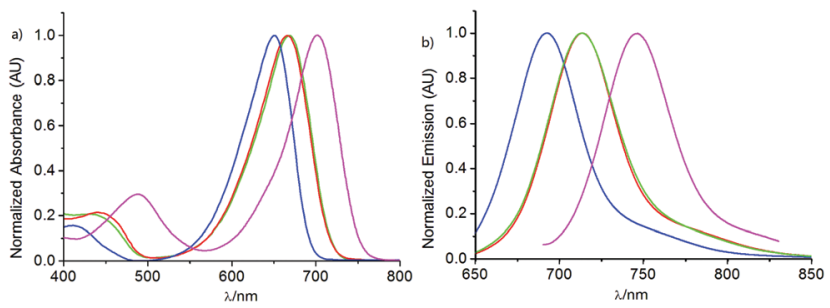

Fig. 3 (a) Normalized absorption and (b) fluorescence spectra of DPh-tBuazaBDP (blue curve), Flu-tBuazaBDP (green), S-tBuazaBDP (red) and S-PhazaBDP (pink) in $\mathrm{CH}_{2} \mathrm{Cl}_{2}$ at $298 \mathrm{~K}$.
Table 1 Optical properties of aza-BODIPYs in $\mathrm{CH}_{2} \mathrm{Cl}_{2}$ at $298 \mathrm{~K}$

\begin{tabular}{|c|c|c|c|c|c|c|}
\hline Dye & $\begin{array}{l}\lambda_{\mathrm{abs}} / \lambda_{\mathrm{em}} \\
{[\mathrm{nm}]}\end{array}$ & $\begin{array}{l}\text { Stokes } \\
\text { shift } \\
{[\mathrm{nm}]}\end{array}$ & $\begin{array}{l}\text { FWHM } \\
{[\mathrm{nm}]}\end{array}$ & $\varepsilon\left[\mathrm{M}^{-1} \mathrm{~cm}^{-1}\right]$ & $\Phi_{\mathrm{f}}$ & $\Phi_{\Delta}$ \\
\hline DPh-tBuazaBDP & $650 / 693$ & 43 & 69 & 83000 & 0.13 & 0.06 \\
\hline Flu-tBuazaBDP & $668 / 713$ & 45 & 74 & 84000 & 0.12 & 0.04 \\
\hline S-tBuazaBDP & $666 / 713$ & 47 & 73 & 85000 & 0.04 & 0.05 \\
\hline S-PhazaBDP & $702 / 746$ & 44 & 52 & 105000 & 0.06 & - \\
\hline
\end{tabular}

DPh-tBuazaBDP with the biphenyl groups, Flu-tBuazaBDP with the luorine substituents by freezing biphenyl rotation has a longer wavelength and higher molar extinction coefficients. Due to non-radiative relaxation by the rotation of the $-t \mathrm{Bu}$ group, both DPh-tBuazaBDP $\left(\Phi_{\mathrm{f}}=0.13\right)$ and Flu-tBuazaBDP (0.12) were found to have low quantum yields, and these are just potentially suitable for phototherapeutic application.

To further gain an insight into the optical difference between DPh-tBuazaBDP and Flu-tBuazaBDP, solvent effect experiments were carried out in solvents ranging from $n$ hexane to DMSO (Fig. S1, S2 and Table S1, ESI $\dagger$ ). The absorption maximum of DPh-tBuazaBDP is $644 \mathrm{~nm}$ in the non-polar solvent $n$-hexane, compared to $658 \mathrm{~nm}$ in the polar solvent DMSO. The absorption maximum is scarely affected by the solvent polarity with all maxima found in a narrow interval of $14 \mathrm{~nm}$, indicating that the solvent-solute interaction is indeed inappreciable in the ground state of DPh-tBuazaBDP. In contrast to the absorption spectra, the increase of the maximum fluorescence peaks and the increase of the half-width of the fluorescence band with increasing solvent polarity were remarkable. The maxima of the fluorescence spectra showed a considerable red-shift $(23 \mathrm{~nm})$ on going from $n$-hexane $(682 \mathrm{~nm})$ to DMSO $(705 \mathrm{~nm})$. But, the full width at half maxima (FWHM) and the molar extinction coefficients in the different solvents were not obviously changed. In Flu-tBuazaBDP, the introduction of the luorine substituents by freezing biphenyl rotation led to a red shift of about $20 \mathrm{~nm}$ and a wider FWHM in various solvents, compared to those of DPh-tBuazaBDP (Fig. 3). However, the Stokes shifts of Flu-tBuazaBDP were smaller than those of DPh-tBuazaBDP.

To unravel the secret of different luminescence properties of S-tBuazaBDP and S-PhazaBDP, theoretical simulations were conducted based on DFT and TDDFT methods. The calculated electronic spectra of both molecules (Fig. S1 and Table S1, ESI $\dagger$ ) are in accordance with the experimental results, which manifests the reliability of the theoretical results. The primary absorption and emission peaks of S-tBuazaBDP both correspond to the $S_{0}-S_{1}$ transition, which consists of the transition between the highest occupied molecular orbital (HOMO) and the lowest unoccupied molecular orbital (LUMO). As shown in Fig. 4, the electron density of the HOMO is mainly distributed on non- $t \mathrm{Bu}$ groups, whereas that of the LUMO is mainly located on the core of the BODIPY, which indicates that the $S_{0}-S_{1}$ transition has a slight charge-transfer property. Similar to S-tBuazaBDP, the primary absorption and emission peaks of S-PhazaBDP also correspond to the $\mathrm{S}_{0}-\mathrm{S}_{1}$ transition, which is 


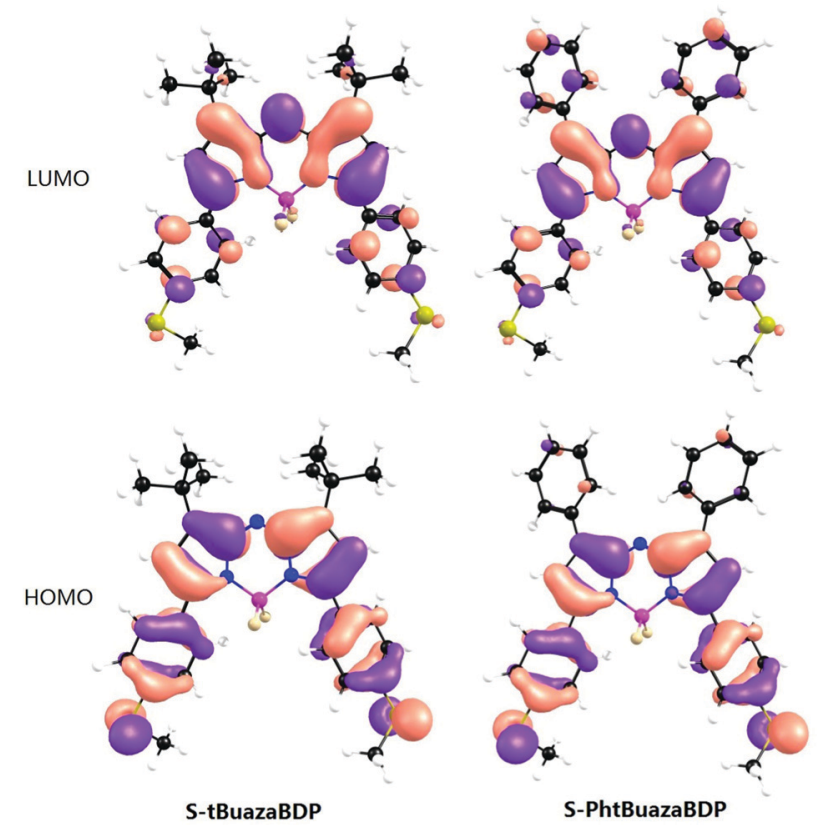

Fig. 4 Frontier molecular orbitals of S-tBuazaBDP and S-PhazaBDP at the B3LYP/6-31 G(d) level with Gaussian 09. LUMO/HOMO $(\mathrm{eV})=-2.32 /$ -3.95 for S-tBuazaBDP; LUMO/HOMO (eV) $=-2.47 /-3.99$ for S-PhazaBDP

contributed by the HOMO-LUMO transition. Different from S-tBuazaBDP, both HOMO and LUMO orbitals of S-PhazaBDP show a $\pi$ electron distribution on the top phenyl group. The elongated conjugation system leads to red shift of the absorption and emission peak of S-PhazaBDP.

The molecular rotation can be restrained in viscous media, and the corresponding fluorescence enhancement could be observed. ${ }^{79}$ However, the emission intensities of S-tBuazaBDP were not sensitive to solvent viscosity (Fig. S4, ESI $\dagger$ ), indicating that the $-t \mathrm{Bu}$ rotation is not strictly limited. Moreover, to reveal the better photothermal effect of S-tBuazaBDP, the rotation process of the top substituted groups of S-tBuazaBDP and S-PhazaBDP was compared, as shown in Fig. 5. The scan process covers $180^{\circ}$ rotation divided into 6 steps. The small energy barrier for the $-t \mathrm{Bu}$ group rotation of S-tBuazaBDP, $2.1 \mathrm{kcal} \mathrm{mol}^{-1}$, suggests its free rotation in solution, which causes more system energy to be released through the nonradiative way. In comparison, the $-\mathrm{Ph}$ group rotation of S-PhazaBDP is more difficult in view of the higher energy barrier of $4.2 \mathrm{kcal} \mathrm{mol}^{-1}$, which may be attributed to the conjugation between phenyl parts and the pyrrole segment in S-PhazaBDP (Scheme 1).

PDT is recognized as a clinical noninvasive modality for the treatment of solid tumors, and the photosensitizer is the critical component of PDT. To gain further insight into singlet oxygen generation of tBuazaBDP dyes, experiments were carried out. tBuazaBDPs in toluene were irradiated with monochromatic light at $650 \mathrm{~nm}$ by using a $150 \mathrm{~W}$ xenon lamp at $0.5 \mathrm{~mW} \mathrm{~cm}^{-2}$. By utilizing 1,3-diphenylisobenzofuran (DPBF) as the singlet oxygen indicator, the efficiency of singlet oxygen
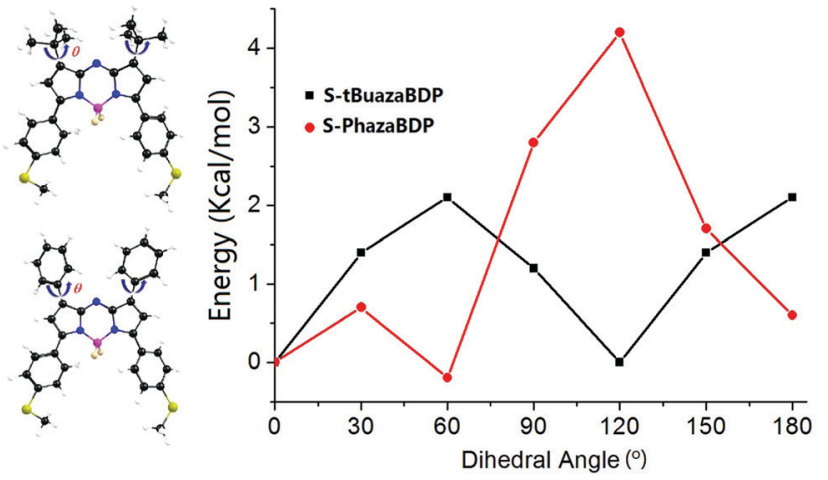

Fig. 5 Energy levels of the $S_{0}$ states of the chemical bond for S-tBuazaBDP and S-PhazaBDP with the dihedral angle $\theta$.

generation was evaluated by detecting the decrease of DPBF absorbance at $416 \mathrm{~nm}$ (Fig. 6a and Fig. S2, S3, ESI $\dagger$ ). According to the linear relationship of the decay curves (Fig. 6b), the ${ }^{1} \mathrm{O}_{2}$ quantum yields of Flu-tBuazaBDP, S-tBuazaBDP and DPh-tBuazaBDP were calculated to be $0.04,0.05$ and 0.06 respectively, which are slightly higher than that $(0.02)$ of the reported dye tetraphenyl aza-BODIPY (Fig. S4, ESI $\dagger$ ). ${ }^{80}$ These results indicated that attaching the $-t \mathrm{Bu}$ groups at 1,7positions in the aza-BODIPY system is still a slightly effective strategy to enhance the singlet oxygen generation. No photobleaching of such dyes was observed during these experiments.

The self-assembly method of dye S-tBuazaBDP and the hydrophilic polymer poloxamer (F127) into nanoparticles was employed (Fig. 7a and b). S-tBuazaBDP-NPs were water soluble (Fig. 7d). The morphology and size of the nanoparticles were measured by utilizing transmission electron microscopy (TEM). As depicted in Fig. 7b, S-tBuazaBDP-NPs were spherical nanoparticles with a diameter below $110 \mathrm{~nm}$. The polydispersity experiments were performed by using dynamic light scattering (DLS). As illustrated in Fig. 7a, the mean hydrodynamic diameter and polydispersity (PDI) of S-tBuazaBDP-NPs were about $50.23 \mathrm{~nm}$ and 0.186. There was no precipitation after S-tBuazaBDP-NPs were placed in water for ten days, suggesting that the nanoparticles have favourable stability (Fig. 7c and d). In the absorption spectra, we can perceive that the maximum absorption wavelength of S-tBuazaBDP-NPs produced a significant red-shift effect and showed a broader peak than
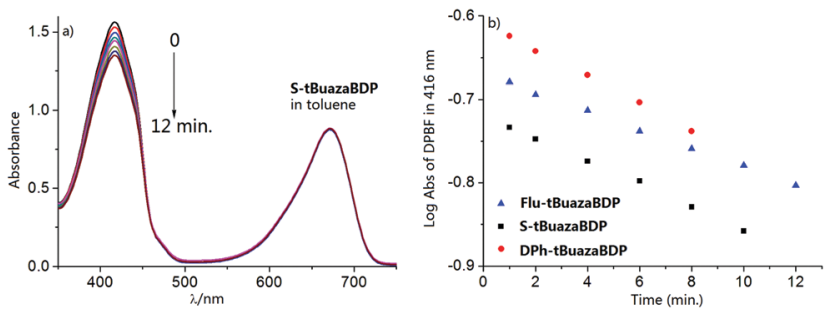

Fig. 6 (a) Time-dependent photodegradation of $6 \times 10^{-5} \mathrm{M}$ DPBF with $8 \mu$ M S-tBuazaBDP. (b) DPBF degradation rate curves with Flu-tBuazaBDP $(S=0.0114)$, S-tBuazaBDP $(S=0.0141)$ and DPh-tBuazaBDP $(S=0.0166)$ in toluene, respectively. 

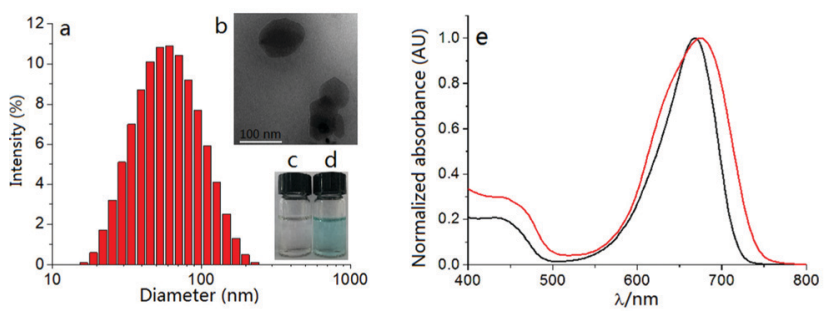

Fig. 7 (a) DLS and (b) TEM of $20 \mu M$ S-tBuazaBDP-NPs in water. (c) Photo of pure water. (d) Photo of S-tBuazaBDP-NPs in water. (e) Normalized absorption of S-tBuazaBDP (black curve) in $\mathrm{CH}_{2} \mathrm{Cl}_{2}$ and S-tBuazaBDP NPs (red curve) in water.

S-tBuazaBDP in $\mathrm{CH}_{2} \mathrm{Cl}_{2}$ (Fig. 7e), which is attributed to the $\pi-\pi$ stacking interaction.

The photothermal conversion effect of S-tBuazaBDP-NPs was further investigated. When employing a $690 \mathrm{~nm}$ laser $\left(0.8 \mathrm{~W} \mathrm{~cm}^{-2}\right)$ as a light source to irradiate S-tBuazaBDP-NPs in water solutions with different concentrations (20, 40 and $80 \mu \mathrm{M}$ ) (Fig. 8b), the temperature of the S-tBuazaBDP-NPs solution significantly increased along with the light irradiation time and concentrations. For instance, the temperature of S-tBuazaBDP-NPs can be rapidly increased by $8.1{ }^{\circ} \mathrm{C}$ after 1 min light irradiation, and the temperature can reach 48.3 ${ }^{\circ} \mathrm{C}$ after 5 min light irradiation, which is beneficial for photothermal therapy in biological systems (Fig. 8a and b). As shown in Fig. S5 (ESI $\dagger$ ), it can be clearly seen that the temperature of the blank solution almost does not change $(\Delta T: 0.9-$ $\left.1.1{ }^{\circ} \mathrm{C}\right)$ under light irradiation. These results showed that S-tBuazaBDP-NPs have a good potential capacity for photothermal therapy. Moreover, when we adjust the $690 \mathrm{~nm}$ laser at different power densities $\left(0.4,0.6\right.$ and $\left.0.8 \mathrm{~W} \mathrm{~cm}^{-2}\right)$ to irradiate $80 \mu \mathrm{M}$ S-tBuazaBDP-NPs, we found that the solution temperature gradually increased with the increase of power density, indicating that the photothermal effect of S-tBuazaBDP-NPs
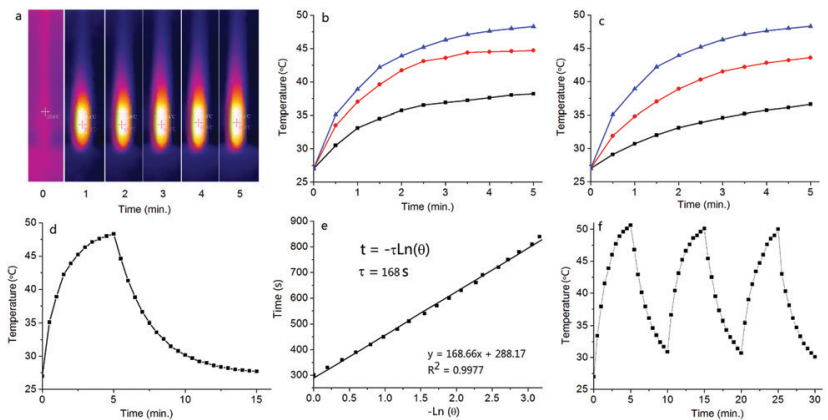

Fig. 8 (a) Photothermal images $\left(27-48.3^{\circ} \mathrm{C}\right)$ of S-tBuazaBDP-NPs in water under $690 \mathrm{~nm}$ laser irradiation $\left(0.8 \mathrm{~W} \mathrm{~cm}^{-2}, 5 \mathrm{~min}\right)$. (b) Photothermal conversion of S-tBuazaBDP-NPs at different concentrations $(20,40$ and $80 \mu \mathrm{M})$ under $690 \mathrm{~nm}$ laser irradiation $\left(0.8 \mathrm{~W} \mathrm{~cm}^{-2}\right)$. (c) Photothermal conversion of S-tBuazaBDP-NPs $(80 \mu \mathrm{M})$ under $690 \mathrm{~nm}$ laser irradiation with different exposure intensity $\left(0.4,0.6\right.$ and $\left.0.8 \mathrm{~W} \mathrm{~cm}^{-2}\right)$. (d) Photothermal response curves of S-tBuazaBDP-NPs aqueous solutions ( $80 \mu \mathrm{M})$ under irradiation and after naturally cooling to room temperature. (e) Linear fitting of $-\operatorname{Ln} \theta$ and time. (f) Photothermal stability study of S-tBuazaBDP-NPs during three heating-cooling cycles. was also power density-dependent (Fig. 8c). To study the photothermal conversion efficiency, a photothermal generation-dissipation curve was recorded and depicted, based on the temperature changes of the heating and cooling curves (Fig. 8d and e). Then, the photothermal conversion efficiency of S-tBuazaBDP-NPs was calculated to be $49 \%$, which is near to the highest one $(50.5 \%)$ in the aza-BODIPY system. ${ }^{81}$ Next, in order to evaluate the photothermal stability of S-tBuazaBDP-NPs, we used a $690 \mathrm{~nm}$ laser $\left(0.8 \mathrm{~W} \mathrm{~cm}^{-2}\right)$ to irradiate the dyes in aqueous solution $(80 \mu \mathrm{M})$ for $5 \mathrm{~min}$ and then cooled them naturally, and the temperature changes were recorded during the process of heating-cooling cycles. As shown in Fig. 8f, the solution temperature increased gradually under light irradiation; upon stopping irradiation, the solution temperature began to decrease gradually. Three heating-cooling cycles were conducted, and the temperature variation was negligible (Fig. 8f), suggesting that S-tBuazaBDP-NPs have excellent thermal stability.

For Flu-tBuazaBDP NPs, the temperature of the solution reached $47.6{ }^{\circ} \mathrm{C}$ after 5 min of illumination $\left(0.8 \mathrm{~W} \mathrm{~cm}^{-2}\right)$ (Fig. S6, ESI $\dagger)$, but was slightly less than that $\left(48.3{ }^{\circ} \mathrm{C}\right)$ of S-tBuazaBDP-NPs. The photothermal effect of Flu-tBuazaBDPNPs was also optical density- and concentration-dependent. According to the temperature changes of the heating and cooling curves (Fig. S6d, e, ESI $\dagger$ ), the photothermal conversion efficiency of Flu-tBuazaBDP-NPs was calculated to be $47 \%$, which is comparable to that $(35-50.5 \%)$ of the reported azaBODIPY-based PTAs. ${ }^{60}$

MTT assay was conducted to further assess the effects of light irradiation and S-tBuazaBDP-NPs concentrations on human colon cancer cell (LoVo) viability. As shown in Fig. S7 (ESI $\dagger$ ), the results showed that $\mathbf{S}$-tBuazaBDP-NPs have low darktoxicity and high photo-toxicity. To visualize the effect of phototherapeutic performance of S-tBuazaBDP-NPs, live-dead cell staining was carried out. After human colon cancer cells were cultured to a certain density, cells in different dishes were treated with the control group, the sole light treatment group, the sole dye treatment group, and dye treatment followed by light irradiation. A $635 \mathrm{~nm}$ NIR laser $\left(10 \mathrm{~mW} \cdot \mathrm{cm}^{-2}, 20 \mathrm{~min}\right)$ was employed as the light source, and the S-tBuazaBDP-NPs concentration was $20 \mu \mathrm{M}$. AM indicated living cells (green fluorescence) and PI indicated dead cells (red fluorescence). From laser confocal microscope imaging observation, the initial three groups showed significant green fluorescence (Fig. 9), and almost no red fluorescence was observed. Once light irradiation was applied after S-tBuazaBDP-NPs were added to cells, red fluorescence was obviously observed, and almost no green fluorescence was observed. In addition, the bright field imaging revealed that the cancer cells became round and showed an obvious state of death, while the other three groups of cancer cells grew well. Therefore, it can be stated that S-tBuazaBDP-NPs could produce effective heat to kill cancer cells under light irradiation, while the dark toxicity was negligible. According to intracellular phototherapeutic experiments, S-tBuazaBDP-NPs had good biocompatibility, low dark-toxicity, and the ability of effectively killing cancer cells and inhibiting 


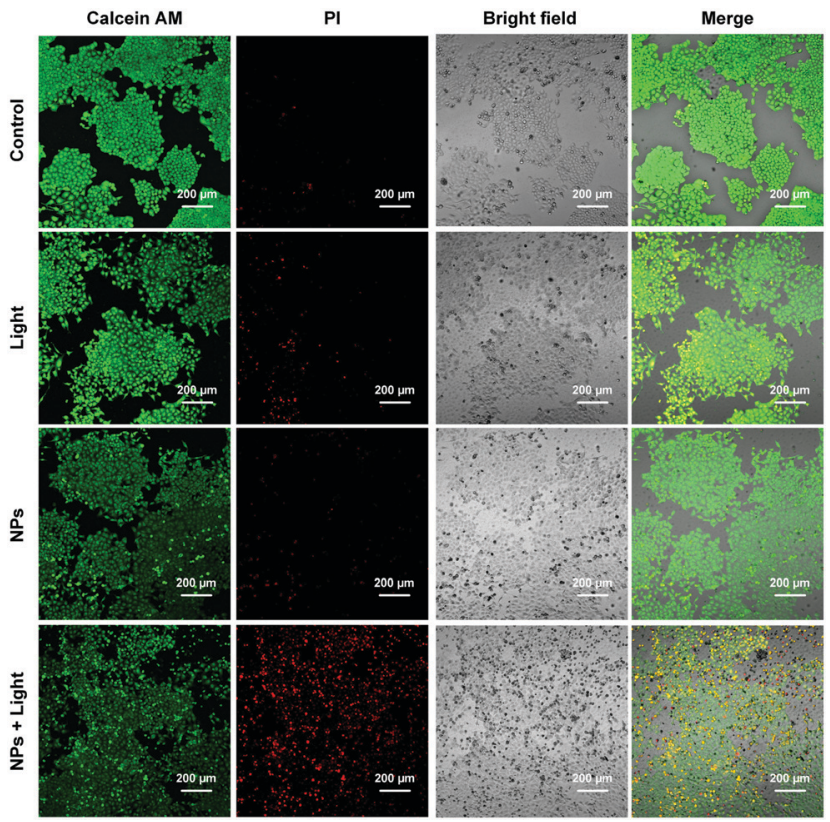

Fig. 9 Calcein AM (green channel) and propidium iodide (PI; red channel) co-staining for live-dead cell imaging after control group, light group, S-tBuazaBDP NPs group and S-tBuazaBDP NPs + light group treatments. A $635 \mathrm{~nm}$ laser $\left(10 \mathrm{~mW} \cdot \mathrm{cm}^{-2}, 20 \mathrm{~min}\right.$ ) was used for light irradiation, dye NPs' concentration was $20 \mu \mathrm{M}$, scale bar: $200 \mu \mathrm{m}$.

the growth of cancer cells under the condition of light irradiation, which are highly desirable for an effective and potential tumor phototherapeutic reagent.

\section{Conclusions}

Using 3,3-dimethylbutan-2-one, aza-BODIPYs with - $t$ Bu groups at 1,7-positions (tBuazaBDPs) were prepared. Based on X-ray crystal analysis, two $-t$ Bu groups in S-tBuazaBDP were found to be located at both sides of the core resulting in a severely twisted structure. tBuazaBDPs with the $-t \mathrm{Bu}$ groups at 1,7positions were indeed blue-shifted, but still locating in the NIR region. The fluorescence quantum yield of S-tBuazaBDP was found to be lower, owing to the ICT effect of the electronrich methylthioether substituents and non-radiative relaxation offered by the rotation of the $-t \mathrm{Bu}$ group. Compared to DPh-tBuazaBDP with the biphenyl groups, Flu-tBuazaBDP with the fluorene substituents by freezing biphenyl rotation has a longer wavelength and higher molar extinction coefficients. The electron density of the HOMO is mainly distributed on non- $t$ Bu groups in S-tBuazaBDP, whereas that of the LUMO is mainly located on the core of aza-BODIPY, indicating that the $\mathrm{S}_{0}-\mathrm{S}_{1}$ transition has a slight charge-transfer property. Different from S-tBuazaBDP, both HOMO and LUMO orbitals of S-PhazaBDP show a $\pi$ electron distribution on the top phenyl group. Experimental results and theoretical calculations proved that the distal $-t \mathrm{Bu}$ groups in S-tBuazaBDP can undergo a lowbarrier rotation. The ${ }^{1} \mathrm{O}_{2}$ quantum yields $(0.04-0.06)$ of tBuazaBDPs were calculated to be low, but are slightly higher than that (0.02) of the classical dye tetraphenyl aza-BODPY. According to the temperature changes of the heating and cooling curves, the photothermal conversion efficiency of S-tBuazaBDP-NPs was calculated to be $49 \%$, which is near to the highest one $(50.5 \%)$ in the aza-BODIPY system. S-tBuazaBDP-NPs had good biocompatibility, low darktoxicity, and the ability of effectively killing cancer cells and inhibiting the growth of cancer cells under the condition of light irradiation, which are highly desirable for an effective and potential tumor phototherapeutic reagent.

\section{Conflicts of interest}

There are no conflicts to declare.

\section{Acknowledgements}

This work was supported by the National Natural Science Foundation of China (22078201, U1908202), "Chunhui Program" cooperative research project of Education Ministry, Natural Science Foundation of Liaoning (2021-NLTS-12-06), Liaoning BaiQianWan Talents Program, serving local project of Education Department of Liaoning (LZ2020005) and Distinguished Professor Project of Liaoning (20183532). We thank Prof. Yohsuke Yamamoto and Dr Rong Shang (Hiroshima University) for their help.

\section{Notes and references}

1 M. A. T. Rogers, Nature, 1943, 151, 504-505.

2 A. Loudet and K. Burgess, Chem. Rev., 2007, 107, 4891-4932.

3 G. Ulrich, A. Harriman and R. Ziessel, Angew. Chem., Int. Ed., 2008, 47, 1184-1201.

4 T. Rappitsch and S. M. Borisov, Chem. - Eur. J., 2021, 27, 10685-10692.

5 S. I. Reja, M. Minoshima, Y. Hori and K. Kikuchi, Chem. Sci., 2021, 12, 3437-3447.

6 X. Li, S. Kolemen, J. Yoon and E. U. Akkaya, Adv. Funct. Mater., 2017, 1604053.

7 F. W. Dagnaw, Y. Cai and Q. Song, Dyes Pigm., 2021, 189, 109257.

8 H. Klfout, A. Stewart, M. Elkhalifa and H. He, ACS Appl. Mater. Interfaces, 2017, 46, 39873-39889.

9 Y. Cakmak, S. Kolemen, S. Duman, G. Y. Dede, Y. Dolen, B. Kilic, Z. Kostereli, L. Tatar-Yildirim, A. L. Dogan, D. Guc and E. U. Akkaya, Angew. Chem., Int. Ed., 2011, 50, 11937-11941.

10 H. Zhu, P. Cheng, P. Chen and K. Pu, Biomater. Sci., 2018, 6, 746-765.

11 Y. Dong, B. Dick and J. Zhao, Org. Lett., 2020, 22, 5535-5539.

12 J. Jiménez, R. Prieto-Montero, B. L. Maroro, F. M. Jiménez, M. J. Ortiz, A. Oliden-Sánchez, Í. López-Arbeloa, V. MartínezMartínez and S. M. Cerero, Chem. - Eur. J., 2020, 22, 601-605.

13 Y. Dong, B. Dick and J. Zhao, Org. Lett., 2020, 22, 5535-5539. 
14 A. Gorman, J. Killoran, C. O'Shea, T. Kenna, W. M. Gallagher and D. F. O'Shea, J. Am. Chem. Soc., 2004, 126, 10619-10631.

15 V. Nguyen, Y. Yan, J. Zhao and J. Yoon, Acc. Chem. Res., 2021, 54, 207-220.

16 G. Li, X. Zhang, W. Zhao, F. Li, K. Xiao, Q. Yu, S. Liu and Q. Zhao, ACS Appl. Mater. Interfaces, 2020, 12, 20180-20190.

17 M. Kang, H. Liu and J. C. Kah, J. Mater. Chem. B, 2020, 47, 10812-10824.

18 J. Feng, W. Ren, F. Kong and Y. Dong, Inorg. Chem. Front., 2021, 8, 848-879.

19 Z. Shi, X. Han, W. Hu, H. Bai, B. Peng, L. Ji, Q. Fan, L. Li and W. Huang, Chem. Soc. Rev., 2020, 49, 7533-7567.

20 H. S. Jung, J. H. Lee, K. Kim, S. Koo, P. Verwilst, J. L. Sessler, C. Kang and J. S. Kim, J. Am. Chem. Soc., 2017, 139, 9972-9978.

21 J. H. Zou, P. Wang, Y. Wang, G. Y. Liu, Y. W. Zhang, Q. Zhang, J. J. Shao, W. L. Si, W. Huang and X. C. Dong, Chem. Sci., 2019, 10, 268-276.

22 X. Miao, W. Hu, T. He, H. Tao, Q. Wang, R. F. Chen, L. Jin, H. Zhao, X. Lu, Q. Fan and W. Huang, Chem. Sci., 2019, 10, 3096-3102.

23 W. Hu, X. Miao, H. Tao, A. Baev, C. Ren, Q. Fan, T. He, W. Huang and P. N. Prasad, ACS Nano, 2019, 13, 12006-12014.

24 M. J. Schnermann, Nature, 2017, 551, 176-177.

25 W. Sun, S. Guo, C. Hu, J. Fan and X. Peng, Chem. Rev., 2016, 116, 7768-7817.

26 X. Zhao, Q. Yao, S. Long, W. Chi, Y. Yang, D. Tan, X. Liu, H. Huang, W. Sun, J. Du, J. Fan and X. Peng, J. Am. Chem. Soc., 2021, 143, 12345-12354.

27 S. Liu, X. Zhou, H. Zhang, H. Ou, J. W. Y. Lam, Y. Liu, L. Shi, D. Ding and B. Z. Tang, J. Am. Chem. Soc., 2019, 141, 5359-5368.

28 S. Sasaki, G. P. C. Drummen and G. Konishi, J. Mater. Chem. C., 2016, 4, 2731-2743.

29 X.-D. Jiang, T. Fang, X. Liu and D. Xi, Eur, J. Org. Chem., 2017, 5074-5079.

30 Y. Liu, N. Song, L. Chen, S. Liu and Z. Xie, Chem. - Asian J., 2018, 16, 989-995.

31 Y. Zhang, Z. Yang, X. Zheng, L. Chen and Z. Xie, J. Mater. Chem. B, 2020, 8, 5305-5311.

32 G. Feng, G. Zhang and D. Ding, Chem. Soc. Rev., 2020, 49, 8179-8234.

33 Y. Xu, T. Feng, T. Yang, H. Wei, H. Yang, G. Li, M. Zhao, S. Liu, W. Huang and Q. Zhao, ACS Appl. Mater. Interfaces, 2018, 10, 16299-16307.

34 B. Wang, G. Feng, M. Seifrid, M. Wang, B. Liu and G. C. Bazan, Angew. Chem., Int. Ed., 2017, 56, 16063-16066.

35 W. Chi, Q. Qiao, R. Lee, W. Liu, Y. S. Teo, D. Gu, M. J. Lang, Y. Chang, Z. Xu and X. Liu, Angew. Chem., Int. Ed., 2019, 58, 7073-7077.

36 D. Xi, M. Xiao, J. Cao, L. Zhao, N. Xu, S. Long, J. Fan, K. Shao, W. Sun, X. Yan and X. Peng, Adv. Mater., 2020, 1907855.

37 H. Wang, H. Zhang, Y. Xiang, W. Pan, N. Li and B. Tang, Nanoscale, 2021, 13, 8790-8794.
38 Q. Zou, M. Abbas, L. Zhao, S. Li, G. Shen and X. Yan, J. Am. Chem. Soc., 2017, 139, 1921-1927.

39 D. Wang, M. M. S. Lee, W. Xu, G. Shan, X. Zheng, R. T. K. Kwok, J. W. Y. Lam, X. Hu and B. Z. Tang, Angew. Chem., Int. Ed., 2019, 58, 5628-5632.

40 D. Chen, Q. Tang, J. Zou, X. Yang, W. Huang, Q. Zhang, J. Shao and X. Dong, Adv. Healthc. Mater., 2018, 7, e1701272.

41 A. H. Ashoka, S. H. Kong, B. Seeliger, B. Andreiuk, R. V. Soares, M. Barberio, M. Diana and A. S. Klymchenko, Biomaterials, 2020, 261, 120306.

42 L. Liu, H. He, Z. Luo, H. Zhou, R. Liang, H. Pan, Y. Ma and L. Cai, Adv. Funct. Mater., 2020, 30, 1910176.

43 X. Huang, J. Wu, M. He, X. Hou, Y. Wang, X. Cai, H. Xin, F. Gao and Y. Chen, Mol. Pharmaceutics, 2019, 16, 2172-2183.

44 J. Zhou, Y. Jiang, S. Hou, P. K. Upputuri, D. Wu, J. Li, P. Wang, X. Zhen, M. Pramanik, K. Pu and H. Duan, ACS Nano, 2018, 12, 2643-2651.

45 Z. Zhang, W. Xu, M. Kang, H. Wen, H. Guo, P. Zhang, L. Xi, K. Li, L. Wang, D. Wang and B. Tang, Adv. Mater., 2020, 32, 2003210.

46 J. Huang, Y. Huang, Z. Xue and S. Zeng, Biomaterials, 2020, 262, 120346.

47 H. Chen, H. Cheng, Q. Dai, Y. Cheng, Y. Zhang, D. Li, Y. Sun, J. Mao, K. Ren, C. Chu and G. Liu, J. Controlled Release, 2020, 323, 635-643.

48 A. M. Gobin, E. M. Watkins, E. Quevedo, V. L. Colvin and J. L. West, Small, 2010, 6, 745-752.

49 D. Wang, Z. Guo, J. Zhou, J. Chen, G. Zhao, R. Chen, M. He, Z. Liu, H. Wang and Q. Chen, Small, 2015, 11, 5956-5967.

50 H. Bi, Y. Dai, J. Xu, R. Lv, F. He, S. Gai, D. Yang and P. Yang, J. Mater. Chem. B, 2016, 4, 5938-5946.

51 S. Zhang, K. Yang and Z. Liu, Sci. China: Chem., 2010, 53, 2217-2225.

52 Y. Jiao, K. Liu, G. Wang, Y. Wang and X. Zhang, Chem. Sci., 2015, 6, 3975-3980.

53 S. Ye, J. Rao, S. Qiu, J. Zhao, H. He, Z. Yan, T. Yang, Y. Deng, H. Ke, H. Yang, Y. Zhao, Z. Guo and H. Chen, Adv. Mater., 2018, 30, 1801216.

54 J. Zhou, Y. Jiang, S. Hou, P. K. Upputuri, D. Wu, J. Li, P. Wang, X. Zhen, M. Pramanik, K. Pu and H. Duan, ACS. Nano., 2018, 12, 2643-2651.

55 Q. Zou, M. Abbas, L. Zhao, S. Li, G. Shen and X. Yan, J. Am. Chem. Soc., 2017, 139, 1921-1927.

56 B. Guo, G. Feng, P. N. Manghnani, X. Cai, J. Liu, W. Wu, S. Xu, X. Cheng, C. Teh and B. Liu, Small, 2016, 12, 6243-6254.

57 C. S. Jin, J. F. Lovell, J. Chen and G. Zheng, ACS Nano, 2013, 7, 2541-2550.

58 S. Su, Y. Ding, Y. Li, Y. Wu and G. Nie, Biomaterials., 2016, 80, 169-178.

59 P. Liang, Q. Tang, Y. Cai, G. Liu, W. Si, J. Shao, W. Huang, Q. Zhang and X. Dong, Chem. Sci., 2017, 8, 7457-7463.

60 J. Y. Liu, P. Bhattarai, Z. Dai and X. Chen, Chem. Soc. Rev., 2019, 48, 2053-2108.

61 L. Wang and C. Niu, J. Mater. Chem. B, 2021, 9, 4079-4097. 62 L. Li, X. Han, M. Wang, C. Li, T. Jia and X. Zhao, Chem. Eng. J., 2021, 417, 128844. 
63 J. Han and K. Burgess, Chem. Rev., 2010, 110, 2709-2728.

64 X. Miao, W. Hu, T. He, H. Tao, Q. Wang, R. F. Chen, L. Jin, H. Zhao, X. Lu, Q. Fan and W. Huang, Chem. Sci., 2019, 10, 3096-3102.

65 Q. Yu, X. Huang, T. Zhang, W. Wang, D. Yang, J. Shao and X. Dong, Chem. Res. Chin. Univ., 2021, 37, 951-959.

66 Y. Xu, T. Feng, T. Yang, H. Wei, H. Yang, G. Li, M. Zhao, S. Liu, W. Huang and Q. Zhao, ACS Appl. Mater. Interfaces, 2018, 10, 16299-16307.

67 J. Yin, X. Jiang, G. Sui, Y. Du, E. Xing, R. Shi, C. Gu, X. Wen, Y. Feng, Z. Shan and S. Meng, J. Mater. Chem. B, 2021, 9, 7461-7471.

68 W. Zhang, L. Yu, Y. Jiang and C. Guo, Biomater. Sci., 2021, 9, 5302-5318.

69 D. Wu and D. F. O'Shea, Org. Lett., 2013, 15, 3392-3395.

70 X.-D. Jiang, D. Xi, J. Zhao, H. Yu, G. Sun and L. Xiao, $R S C$ Adv., 2014, 4, 60970-60973.

71 X.-D. Jiang, S. Li, B. L. Guennic, D. Jacquemin, D. Escudero and L. Xiao, Phys. Chem. Chem. Phys., 2016, 18, 32686-32690.

72 T. Fang, X.-D. Jiang, C. Sun and Q. Li, Sen. Actuators, B, 2019, 290, 551-557.
73 X.-D. Jiang, Y. Toya, S. Matsukawa, S. Kojima, J. O. C. JimenezHalla, R. Shang, M. Nakamoto and Y. Yamamoto, Chem. Sci., 2019, 10, 3466-3472.

74 C. Li, H. Ge, D. Zhang, C. Sun, S. Yue, X.-D. Jiang and J. Du, Sen. Actuators, B, 2021, 344, 130213.

75 Z. Otwinowski and W. Minor, Methods Enzymol., 1997, 276, 307-326.

76 G. M. Sheldrick, SHELX-97, University of Göttingen, Göttingen, Germany, 1997.

77 M. J. Frisch, G. W. Trucks, H. B. Schlegel, G. E. Scuseria and M. A. Robb, et al., Gaussian 09W, revision A.1., Gaussian Inc., Wallingford, CT, 2009.

78 X.-D. Jiang, X. Liu, T. Fang and C. Sun, Dyes Pigm., 2017, 146, 438-444.

79 W. Chi, Q. Qiao, R. Lee, W. Liu, Y. S. Teo, D. Gu, M. J. Lang, Y. Chang, Z. Xu and X. Liu, Angew. Chem., Int. Ed., 2019, 58, 7073-7077.

80 Z. Yu, J. Zhou, X. Ji, G. Lin, S. Xu, X. Dong and W. Zhao, J. Med. Chem., 2020, 63, 9950-9964.

81 Y. Xu, S. Wang, Z. Chen, R. Hu, S. Li, Y. Zhao, L. Liu and J. Qu, J. Nanobiotechnol., 2021, DOI: 10.1186/s12951-021-00782-y. 